\title{
On the Instantaneous Acceleration of Points in a Rigid Body
}

\author{
J.M. Selig \\ London South Bank University \\ London SE1 OAA, U.K.
}

\begin{abstract}
This work re-examines some classical results in the kinematics of points in space using modern vector-matrix methods. In particular, some very simple Lie theory allows the velocities and accelerations of points to be found in terms of the instantaneous twist of the motion and its derivative. From these results many of the classical results follow rather simply.

Although most of the results are well known, some new material is presented. In particular, the discriminant curve that separates cases with one or three real acceleration axes is found and plotted. Another new result concerns the chords to the cubic of inflection points. It is shown that for points on such a chord the osculating planes of the point's trajectories are parallel. Also a new result is found which distinguishes between cases where the Bresse hyperboloid of points whose velocities and accelerations are perpendicular, has one or two sheets.
\end{abstract}

Keywords:

Spatial motion, spatial kinematics, acceleration, points of inflection 2000 MSC: 70B10

\section{Introduction}

The advent of cheap solid-state accelerometers has made it possible to incorporate these devices into everyday consumer electronic products such as mobile phones and games controllers. This has prompted interest in the

Email address: seligjm@lsbu.ac.uk (J.M. Selig) 
problem of finding the state of motion of a rigid body from measurements of its acceleration at a finite number of points, see for example [1].

In this work an older problem is re-examined, the problem of describing the instantaneous acceleration field of a rigid-body motion. This is essentially instantaneous kinematics. It is expected that a better understanding of the acceleration and velocity fields of a rigid motion will illuminate the problem mentioned above. A better understanding of this area may also help the study of finite rigid-body motions. This is an area that has several applications to robot path planning and computer animation. It has also long been appreciated that instantaneous kinematics is of fundamental importance to the theory of mechanisms.

Since this is a very old and well studied area many of the results are well known. The aim of this present work is first to give modern proofs of these well known results and then to extend them where possible. Further, by putting these ideas into a common geometrical framework, relations between different results can be seen. Perhaps the standard reference in this area is the seminal work of Bottema and Roth, [2]. However, over the years many other workers have revisited this area, see for example, [3], [4], [5].

Due to limitations of space, only general results are considered in this work. However, there are many special cases where curves and surfaces degenerate or acquire special properties. Many of these special cases are studied in [6], but see also [7].

The first result addressed in this work is the existence and location of the acceleration centre of the motion, this was studied in [8]. Here simple matrix methods are used to quickly derive familiar results. A key method used here is a series of symbolic relations for certain $3 \times 3$ matrices derived in full in the appendix to this work.

Next, the acceleration axes are studied. A novel result here is the explicit determination of the discriminant curve which distinguishes the cases where there are one or three real acceleration axes.

The following section concerns the acceleration ellipsoids as studied in [9]. The novelty of this section is the relationship between the principal axes of the ellipsoids and the singular value decomposition of the angular acceleration tensor. This tensor was also studied in [10]. Conditions for the ellipsoids to be cylindrical are found.

The complex of acceleration lines are studied in the following section. The same methods used in the preceding sections allow a very simple and explicit derivation of this tetrahedral quadratic line complex. 
In the rest of the work the acceleration and velocity of points are considered together. The main results in this area is that the points in the body that are instantaneously at points of inflection form a twisted cubic curve. This result seems to date back to [11], but has been rediscovered by many others over the years, for example see [12]. Again a simple derivation is given here, this allows an explicit parameterisation of the curve to be found.

In the next section, what is believed to be a completely novel result is presented. This relates the osculating planes for points, to the chords of the cubic of inflection points. The osculating plane of a point is the plane spanned by the velocity and acceleration vectors at the point.

Another new idea is introduced in the following section. It is well known that the tangent lines to a twisted cubic curve lie in a unique linear line complex. Here the line complex determined by the cubic of inflection points is studied. The unique screw defining the line complex is found.

Finally, the Bresse hyperboloid of points with perpendicular velocity and acceleration is considered. This has been studied previously in [13], for example. Here the centre of the hyperboloid is found and the condition for the hyperboloid to have one or two sheets is determined.

To begin, key relations for the velocity and acceleration of points in a moving rigid body are derived.

\section{Velocity and Acceleration of Points}

Let $G(t)$ be a rigid body motion, that is $G(t) \in S E(3)$. In the $4 \times 4$ representation of the group this can be written,

$$
G(t)=\left(\begin{array}{cc}
R(t) & \mathbf{p}(t) \\
0 & 1
\end{array}\right)
$$

where $R$ is a $3 \times 3$ rotation matrix and $\mathbf{p}$ is a translation vector. The parameter $t$ will usually be though of as time in the following.

If $\mathbf{r}_{0}$ is a point on the moving body when $t=0$, then its position at subsequent times $\mathbf{r}(t)$, will be given by,

$$
\left(\begin{array}{c}
\mathbf{r}(t) \\
1
\end{array}\right)=\left(\begin{array}{cc}
R(t) & \mathbf{p}(t) \\
0 & 1
\end{array}\right)\left(\begin{array}{c}
\mathbf{r}_{0} \\
1
\end{array}\right)
$$

The velocity of the point will be found by differentiating with respect to $t$, this will be denoted by a dot, so

$$
\left(\begin{array}{c}
\dot{\mathbf{r}}(t) \\
0
\end{array}\right)=\left(\begin{array}{cc}
\Omega & \mathbf{v} \\
0 & 0
\end{array}\right)\left(\begin{array}{cc}
R(t) & \mathbf{p}(t) \\
0 & 1
\end{array}\right)\left(\begin{array}{c}
\mathbf{r}_{0} \\
1
\end{array}\right)=\left(\begin{array}{cc}
\Omega & \mathbf{v} \\
0 & 0
\end{array}\right)\left(\begin{array}{c}
\mathbf{r}(t) \\
1
\end{array}\right)
$$


where $\Omega$ is the anti-symmetric matrix representing the angular velocity of the body, that is the matrix which satisfies $\Omega \mathbf{r}=\boldsymbol{\omega} \times \mathbf{r}$ for any vector $\mathbf{r}$. The vector $\mathbf{v}$ is a characteristic linear velocity of the motion. It is easily seen that $\mathbf{v}$ is the velocity of the point on the body which is located at the origin at time $t$. In terms 3 -vectors the velocity can be written,

$$
\dot{\mathbf{r}}(t)=\boldsymbol{\omega} \times \mathbf{r}(t)+\mathbf{v} .
$$

The acceleration of the point will be given by the second derivative,

$$
\left(\begin{array}{c}
\ddot{\mathbf{r}}(t) \\
0
\end{array}\right)=\left(\begin{array}{cc}
\dot{\Omega} & \dot{\mathbf{v}} \\
0 & 0
\end{array}\right)\left(\begin{array}{c}
\mathbf{r}(t) \\
1
\end{array}\right)+\left(\begin{array}{cc}
\Omega & \mathbf{v} \\
0 & 0
\end{array}\right)^{2}\left(\begin{array}{c}
\mathbf{r}(t) \\
1
\end{array}\right)
$$

This can be summarised as,

$$
\ddot{\mathbf{r}}(t)=W \mathbf{r}(t)+\mathbf{a}_{0},
$$

where $W=\left(\dot{\Omega}+\Omega^{2}\right)$ is the angular acceleration tensor referred to in [10] and $\mathbf{a}_{0}=\dot{\mathbf{v}}+\Omega \mathbf{v}=\dot{\mathbf{v}}+\boldsymbol{\omega} \times \mathbf{v}$, is the acceleration vector of the point at the origin at time $t$.

In the rest of this work the explicit dependence on $t$ will usually be dropped for brevity. Futher details on this approach to the velocity and acceleration of points can be found in [14].

\section{The Acceleration Centre}

The acceleration centre is the point in space which, at the instant under consideration, has no acceleration. This point will satisfy the matrix equation,

$$
W \mathbf{r}_{c}+\mathbf{a}_{0}=\left(\dot{\Omega}+\Omega^{2}\right) \mathbf{r}_{c}+(\dot{\mathbf{v}}+\boldsymbol{\omega} \times \mathbf{v})=\mathbf{0} .
$$

The acceleration centre $\mathbf{r}_{c}$, is therefore given by,

$$
\mathbf{r}_{c}=-W^{-1} \mathbf{a}_{0}=-\left(\dot{\Omega}+\Omega^{2}\right)^{-1}(\dot{\mathbf{v}}+\boldsymbol{\omega} \times \mathbf{v}) .
$$

Thus a unique acceleration centre exists if and only if the matrix $W$ is non-singular. Using equation (A.1) from the Appendix, it can be seen that this matrix is non-singular when, $-|\boldsymbol{\omega} \times \dot{\boldsymbol{\omega}}|^{2} \neq 0$, that is so long as $\boldsymbol{\omega} \neq \mathbf{0}$, $\dot{\boldsymbol{\omega}} \neq \mathbf{0}$ and $\boldsymbol{\omega}$ is not parallel to $\dot{\boldsymbol{\omega}}$.

Using (A.1) and (A.2), the coordinates of the acceleration centre can be computed explicitly. Before doing this, it is convenient to denote the angular 
acceleration of the body as $\boldsymbol{\alpha}=\dot{\boldsymbol{\omega}}$, to be compatible with previous work. With this notation the equation for the acceleration centre is,

$$
\mathbf{r}_{c}=\frac{\boldsymbol{\alpha}\left(\boldsymbol{\alpha} \cdot \mathbf{a}_{0}\right)+(\boldsymbol{\alpha} \times \boldsymbol{\omega})\left(\boldsymbol{\omega} \cdot \mathbf{a}_{0}\right)-\boldsymbol{\omega}\left((\boldsymbol{\alpha} \times \boldsymbol{\omega}) \cdot \mathbf{a}_{0}\right)+|\boldsymbol{\omega}|^{2} \boldsymbol{\omega}\left(\boldsymbol{\omega} \cdot \mathbf{a}_{0}\right)}{|\boldsymbol{\omega} \times \boldsymbol{\alpha}|^{2}} .
$$

The relation for the vector triple product this can be tidied a little to give,

$$
\mathbf{r}_{c}=\frac{\left(\boldsymbol{\alpha} \cdot \mathbf{a}_{0}\right) \boldsymbol{\alpha}+|\boldsymbol{\omega}|^{2}\left(\boldsymbol{\omega} \cdot \mathbf{a}_{0}\right) \boldsymbol{\omega}+\mathbf{a}_{0} \times(\boldsymbol{\omega} \times(\boldsymbol{\omega} \times \boldsymbol{\alpha}))}{|\boldsymbol{\omega} \times \boldsymbol{\alpha}|^{2}} .
$$

This is in agreement with the results of [8], however these authors choose to write $|\boldsymbol{\omega} \times \boldsymbol{\alpha}|^{2}$ as $|\boldsymbol{\omega}|^{2}|\boldsymbol{\alpha}|^{2}-(\boldsymbol{\omega} \cdot \boldsymbol{\alpha})^{2}$.

\section{The Acceleration Axes}

Consider the set of points in the body with acceleration normal to some given direction. Suppose the given direction is $\mathbf{m}$, then the points sought must satisfy the equation,

$$
0=\mathbf{m} \cdot \ddot{\mathbf{r}}=\mathbf{m}^{T} W \mathbf{r}+\mathbf{m}^{T} \mathbf{a}_{0}
$$

This is the equation of a plane through the acceleration centre. This can be seen from equation (5) above, which shows that the acceleration centre satisfies the equation for any direction $\mathbf{m}$. Notice that the normal to the plane is given by $W^{T} \mathbf{m}$. In general, the normal to the plane will be different from the normal to the acceleration vectors of the points in the plane. However, if $\mathbf{m}$ is an eigenvector of the matrix $W^{T}=\left(\dot{\Omega}+\Omega^{2}\right)^{T}=\left(-\dot{\Omega}+\Omega^{2}\right)$, then the acceleration vectors of points in the corresponding plane will lie in the plane. The matrix can have one or three real eigenvalues, so there are correspondingly one or three such planes. Exceptionally, the matrix can have two real eigenvalues if one of these eigenvalues is repeated.

If there are three real planes then their point of intersection will, of course, be located at the acceleration centre. These planes do not seem to have a particular name in the literature, here they will be labelled $\pi_{12}, \pi_{23}$ and $\pi_{31}$ corresponding to the eigenvalues $\mathbf{m}_{3}, \mathbf{m}_{1}$ and $\mathbf{m}_{2}$ respectively.

Next, consider the sets of points whose acceleration vector lies in a given direction. Suppose the given direction is specified by a vector product, $\mathbf{m}_{1} \times$ 
$\mathbf{m}_{2}$. From the above it is easy to see that the points with acceleration vector in the direction $\mathbf{m}_{1} \times \mathbf{m}_{2}$ lie on the intersection of the two planes,

$$
\mathbf{m}_{1}^{T} W \mathbf{r}+\mathbf{m}_{1}^{T} \mathbf{a}_{0}=0, \quad \text { and } \quad \mathbf{m}_{2}^{T} W \mathbf{r}+\mathbf{m}_{2}^{T} \mathbf{a}_{0}=0 .
$$

That is, along a line through the acceleration centre. Notice that this argument is reversible, the points on any line through the acceleration centre will have acceleration parallel to some direction.

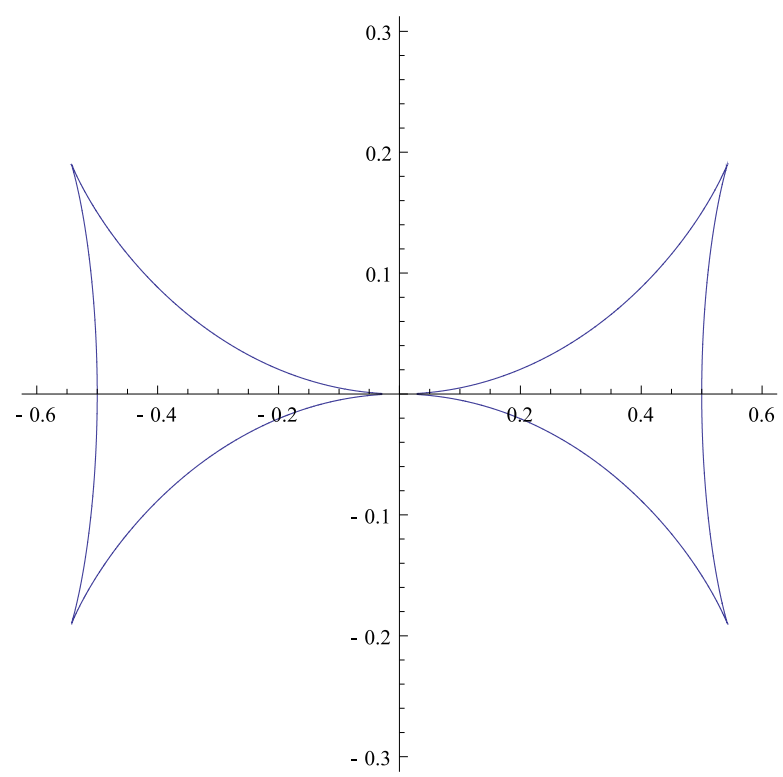

Figure 1: The Discriminant curve. Here $\boldsymbol{\omega}$ is assumed to be of unit length and directed along the vertical axis. The position vector of a point in the plane determines the angular acceleration vector $\dot{\boldsymbol{\omega}}$, the length of the position vector is $|\dot{\boldsymbol{\omega}}|$ the magnitude of the angular acceleration and the angle between the vertical axis and the position vector is $\phi$. Inside the curve the motion has 3 real acceleration axes while outside only one.

Following Bottema and Roth [2, Chap. $6 \S 12]$, consider lines where the acceleration of any point on the line is directed along that line. From the above we can see that if $\mathbf{m}_{1}$ and $\mathbf{m}_{2}$ are eigenvectors of $W^{T}$ then the line through the acceleration centre with direction $\mathbf{m}_{1} \times \mathbf{m}_{2}$ is such a line. Recall that the acceleration of points in the planes through the acceleration centre and normal to $\mathbf{m}_{1}$ or $\mathbf{m}_{2}$ lie in the planes $\pi_{23}$ and $\pi_{31}$. So the intersection of two such planes gives a line of points with acceleration vectors perpendicular to both $\mathbf{m}_{1}$ and $\mathbf{m}_{2}$. These normals are also normal to the line of intersection. In the literature these lines are known as the acceleration axes. 
Using the relation (A.3) given in the Appendix, it is simple to show that if $\mathbf{n}_{1}$ and $\mathbf{n}_{2}$ are eigenvectors of the matrix $W$, then $\mathbf{n}_{1} \times \mathbf{n}_{2}$ is an eigenvector of the transposed matrix $W^{T}$. This justifies the notation introduced above: $\pi_{12}$ is the plane of points whose accelerations are normal to $\mathbf{n}_{1} \times \mathbf{n}_{2}=\mathbf{m}_{3}$. Also the intersection of two such planes $\pi_{12}$ and $\pi_{23}$ say, is the acceleration axis directed along the eigenvector $\mathbf{n}_{2}=\mathbf{m}_{3} \times \mathbf{m}_{1}$.

In fact even when $W^{T}$ only has one real eigenvector, the acceleration axis is directed along the corresponding real eigenvector of $W$. To see this consider the points along a line through the acceleration centre, $\mathbf{r}=\mathbf{r}_{c}+\lambda \mathbf{u}$, where $\mathbf{u}$ is the direction of the line and $\lambda$ is an arbitrary parameter. The acceleration of such a point is given by,

$$
\ddot{\mathbf{r}}=W\left(\mathbf{r}_{c}+\lambda \mathbf{u}\right)+\mathbf{a}_{0}=\lambda W \mathbf{u} .
$$

Now, for this acceleration to be directed along the line we must have,

$$
\mathbf{0}=\mathbf{u} \times \ddot{\mathbf{r}}=U W \mathbf{u},
$$

the parameter $\lambda$ is irrelevant. Clearly, the only solutions are when $\mathbf{u}$ is an eigenvector of $W$. Notice that the acceleration vectors of points on lines not passing through the acceleration centre, do not all share the same direction. So the solutions above are the only possible solutions.

This leads to consideration of the circumstances under which $W=(\dot{\Omega}+$ $\left.\Omega^{2}\right)$ has one, two or three real eigenvalues. To find the characteristic equation of the matrix we can use the same methods as those used in the appendix. The characteristic equation for the normal form is,

$$
\begin{aligned}
\operatorname{det}\left(\begin{array}{ccc}
-b_{z}^{2}-\lambda & -a_{z} & a_{y} \\
a_{z} & -b_{z}^{2}-\lambda & -a_{x} \\
-a_{y} & a_{x} & -\lambda
\end{array}\right)= \\
\quad-\lambda^{3}-2 b_{z}^{2} \lambda^{2}-\left(a_{x}^{2}+a_{y}^{2}+a_{z}^{2}+b_{z}^{4}\right) \lambda-\left(a_{x}^{2}+a_{y}^{2}\right) b_{z}^{2}=0 .
\end{aligned}
$$

If $\mathbf{a}$ is substituted by $\dot{\boldsymbol{\omega}}$ and $\mathbf{b}$ by $\boldsymbol{\omega}$, then writing the result in terms of invariants and cancelling a minus sign produces the equation,

$$
\lambda^{3}+2|\boldsymbol{\omega}|^{2} \lambda^{2}+\left(|\dot{\boldsymbol{\omega}}|^{2}+|\boldsymbol{\omega}|^{4}\right) \lambda+|\dot{\boldsymbol{\omega}} \times \boldsymbol{\omega}|^{2}=0 .
$$

The discriminant of a general cubic equation $a_{3} \lambda^{3}+a_{2} \lambda^{2}+a_{1} \lambda+a_{0}=0$ is given by, $D=a_{1}^{2} a_{2}^{2}-4 a_{0} a_{2}^{3}-4 a_{1}^{3} a_{3}+18 a_{0} a_{1} a_{2} a_{3}-27 a_{0}^{2} a_{3}^{2}$, see [15]. For the 
cubic above, it may be assumed that, $|\dot{\boldsymbol{\omega}} \times \boldsymbol{\omega}|=|\dot{\boldsymbol{\omega}}||\boldsymbol{\omega}| \sin \phi$, where $\phi$ is the angle between the angular velocity and angular acceleration vectors. After some computation the discriminant becomes,

$$
\begin{gathered}
D=-27|\dot{\boldsymbol{\omega}}|^{4}|\boldsymbol{\omega}|^{4} \sin ^{4} \phi+4\left(9|\dot{\boldsymbol{\omega}}|^{2}+|\boldsymbol{\omega}|^{4}\right)|\dot{\boldsymbol{\omega}}|^{2}|\boldsymbol{\omega}|^{4} \sin ^{2} \phi- \\
4\left(|\dot{\boldsymbol{\omega}}|^{4}+2|\dot{\boldsymbol{\omega}}|^{2}|\boldsymbol{\omega}|^{4}+|\boldsymbol{\omega}|^{8}\right)|\dot{\boldsymbol{\omega}}|^{2}
\end{gathered}
$$

The significance of this discriminant is that if $D>0$ the cubic will have 3 real roots, but if $D<0$ it has just one real root. When $D=0$, at least one of the roots is repeated. Notice that the discriminant depends only on the relative magnitudes of the angular velocity and angular acceleration vectors, together with their angular separation. Coordinates can be chosen so that $\boldsymbol{\omega}$ is a unit vector in the $y$-direction and $\dot{\boldsymbol{\omega}}$ lies in the $x y$-plane. With this choice of coordinates the points in the $x y$-plane where $\dot{\boldsymbol{\omega}}$ satisfies $D=0$ can be plotted, see Figure 1. A point on the curve in this figure has a position vector with length $|\dot{\boldsymbol{\omega}}|$ and its angle to the $y$-axis is $\phi$. For angular accelerations outside the curve $D<0$, hence only one acceleration axis exists for these cases. Inside the curve $D>0$, so in these cases there are three acceleration axes. On the curve we expect that there will be two acceleration axes but with one a repeated axis, at the ordinary cusp point on the curve there is a single, thrice repeated, acceleration axis. The location of these cusp point are easily found, they occur when $|\boldsymbol{\omega}|=\sqrt{3}|\dot{\boldsymbol{\omega}}|$ and the angle between the angular velocity and angular acceleration vectors satisfies, $\sin \phi= \pm 2 \sqrt{2} / 3$

\section{Acceleration Ellipsoids}

Bokelberg et al. [9] studied the idea of acceleration ellipsoids. See also [2, Chap. $6 \S 12$. These are surfaces on which all points have acceleration vectors with the same magnitude. Assuming the magnitude of the acceleration is given by $k$, these surfaces can be defined by the following equation,

$$
\ddot{\mathbf{r}}^{T} \ddot{\mathbf{r}}=k^{2}=\left(W \mathbf{r}+\mathbf{a}_{0}\right)^{T}\left(W \mathbf{r}+\mathbf{a}_{0}\right) .
$$

It is easy to see that if coordinates centred on the acceleration centre are used, so that $\mathbf{a}_{0}=\mathbf{0}$, then the equation for the surfaces reduces to,

$$
\mathbf{r}^{T} W^{T} W \mathbf{r}=k^{2}
$$


The symmetric matrix on the left is a Gram matrix and hence is positive definite so long as $W$ is non-singular. This shows that the surfaces are indeed ellipsoids. The axes of these ellipsoids are directed along the eigenvectors of the matrix $W^{T} W$. These are closely related to the singular value decomposition of the matrix $W$. Suppose that $U^{T} W V=\Sigma$ is the singular value decomposition of $W$, so that $U$ and $V$ are orthogonal matrices and $\Sigma$ is the diagonal matrix of singular values. The symmetric matrix $W^{T} W$ can be diagonalised using the matrix $V$, that is,

$$
\left(U^{T} W V\right)^{T}\left(U^{T} W V\right)=\Sigma^{2}=V^{T} W^{T} W V .
$$

So the columns of $V$ are the eigenvectors of $W^{T} W$ and the squares of the singular values of $W$ are the eigenvalues of $W^{T} W$.

The acceleration ellipsoids of an instantaneous motion will be cylindrical if two of the eigenvalues coincide. To investigate this phenomenon the characteristic equation of the matrix $W^{T} W$ can be computed. This is most easily done using the same methods as used in the previous section. The result is,

$$
\lambda^{3}-2\left(|\dot{\boldsymbol{\omega}}|^{2}+|\boldsymbol{\omega}|^{4}\right) \lambda^{2}+\left(|\dot{\boldsymbol{\omega}}|^{2}+|\boldsymbol{\omega}|^{4}\right)^{2} \lambda-|\dot{\boldsymbol{\omega}} \times \boldsymbol{\omega}|^{4}=0 .
$$

Substituting this into the expression for the discriminant of a cubic given in the previous section yields,

$$
D=|\dot{\boldsymbol{\omega}} \times \boldsymbol{\omega}|^{4}\left(4\left(|\dot{\boldsymbol{\omega}}|^{2}+|\boldsymbol{\omega}|^{4}\right)^{3}-27|\dot{\boldsymbol{\omega}} \times \boldsymbol{\omega}|^{4}\right) .
$$

Assuming that the angular velocity and angular acceleration are not parallel or zero, that is that the term $|\dot{\boldsymbol{\omega}} \times \boldsymbol{\omega}|$ does not vanish, we can look for solutions as in the previous section by assuming that the angle between the angular velocity and angular acceleration is $\phi$ so that $|\dot{\boldsymbol{\omega}} \times \boldsymbol{\omega}|=|\dot{\boldsymbol{\omega}}||\boldsymbol{\omega}| \sin \phi$. Now for physical solutions of the equation $D=0$ we must have that $|\sin \phi| \leq 1$ and hence that,

$$
4\left(|\dot{\boldsymbol{\omega}}|^{2}+|\boldsymbol{\omega}|^{4}\right)^{3} \leq 27|\dot{\boldsymbol{\omega}}|^{4}|\boldsymbol{\omega}|^{4}
$$

or

$$
4\left(|\dot{\boldsymbol{\omega}}|^{2}+|\boldsymbol{\omega}|^{4}\right)^{3}-27|\dot{\boldsymbol{\omega}}|^{4}|\boldsymbol{\omega}|^{4} \leq 0 .
$$

This can be solved if we assume that $|\dot{\boldsymbol{\omega}}|=\mu|\boldsymbol{\omega}|^{2}$ for some positive constant $\mu$. Substituting into the equation above and simplifying gives,

$$
\left(4\left(\mu^{2}+1\right)^{3}-27 \mu^{4}\right)|\boldsymbol{\omega}|^{12} \leq 0 .
$$


The polynomial in $\mu$ factorises and the magnitude of the angular velocity is positive so this simplifies to,

$$
\left(1+4 \mu^{2}\right)\left(\mu^{2}-2\right)^{2} \leq 0 .
$$

This is never negative, the only solutions are when the left-hand side of the inequality vanishes, that is when $\mu=\sqrt{2}$. This corresponds to $\sin \phi= \pm 1$ and hence $\phi= \pm(\pi / 2)$, the angular velocity and angular acceleration are perpendicular in this case with $|\dot{\boldsymbol{\omega}}|=\sqrt{2}|\boldsymbol{\omega}|^{2}$. This is the only case where two eigenvalues of $W^{T} W$ are the same, in fact it is easy to compute the eigenvalues here; one is $4|\boldsymbol{\omega}|^{4}$ and the other two have the value $|\boldsymbol{\omega}|^{4}$. In particular note that it is not possible for the acceleration ellipsoids to be spheres. In [2, Chap. $6 \S 12$ ], a relation is also given for the lengths of the semi-axes of these ellipses.

\section{Complex of Acceleration Lines}

Consider the line complex formed by taking lines through points in the direction of the acceleration vector through the point. In [2, Chap. 6 \$12] it is shown that this is a quadratic line complex. Here a simple derivation is given which produces the symmetric matrix defining the complex.

Assume that the Plücker coordinates of the line have the form $\left(\boldsymbol{\psi}^{T}, \mathbf{u}^{T}\right)$. That is the vector $\boldsymbol{\psi}=\left(p_{01}, p_{02}, p_{03}\right)^{T}$, is the direction of the line and $\mathbf{u}=$ $\left(p_{23}, p_{31}, p_{12}\right)^{T}$, is the moment of the line. The direction of the line is given by,

$$
\boldsymbol{\psi}=\ddot{\mathbf{p}}=W \mathbf{p}+\mathbf{a}_{0}=\left(\dot{\Omega}+\Omega^{2}\right) \mathbf{p}+(\dot{\mathbf{v}}+\boldsymbol{\omega} \times \mathbf{v}) .
$$

The moment of the line is given by,

$$
\mathbf{u}=\mathbf{p} \times \boldsymbol{\psi} .
$$

From the relation for the vector product given in (A.3) below we have that,

$$
W \mathbf{p} \times W \boldsymbol{\psi}=\operatorname{Adj}\left(W^{T}\right)(\mathbf{p} \times \boldsymbol{\psi})=\operatorname{Adj}\left(W^{T}\right) \mathbf{u} .
$$

Substituting for $W \mathbf{p}=\boldsymbol{\psi}-\mathbf{a}_{0}$ from (12) above gives,

$$
\operatorname{Adj}\left(W^{T}\right) \mathbf{u}=\left(\boldsymbol{\psi}-\mathbf{a}_{0}\right) \times W \boldsymbol{\psi} .
$$

Now the scalar product of this equation with $\boldsymbol{\psi}$ can be taken, that is the equation can be multiplied on the left by $\boldsymbol{\psi}^{T}$ to get,

$$
\boldsymbol{\psi}^{T} \operatorname{Adj}\left(W^{T}\right) \mathbf{u}=-\boldsymbol{\psi}^{T} A_{0} W \boldsymbol{\psi},
$$


where $A_{0}$ is the $3 \times 3$ antisymmetric matrix corresponding to the vector $\mathbf{a}_{0}$. This is a quadratic equation in the Plücker coordinates and hence defines a quadratic line complex. This can be written as a matrix equation,

$$
\left(\boldsymbol{\psi}^{T}, \mathbf{u}^{T}\right)\left(\begin{array}{cc}
A_{0} W-W^{T} A_{0} & \operatorname{Adj}\left(W^{T}\right) \\
\operatorname{Adj}(W) & 0
\end{array}\right)\left(\begin{array}{l}
\boldsymbol{\psi} \\
\mathbf{u}
\end{array}\right)=0
$$

where the equation has been written using a symmetric matrix by adding the equation above to its transpose. The matrix can be simplified a little by choosing the origin to be at the acceleration centre, so that $\mathbf{a}_{0}=\mathbf{0}$, in these coordinates the equation becomes,

$$
\left(\boldsymbol{\psi}^{T}, \mathbf{u}^{T}\right)\left(\begin{array}{cc}
0 & \operatorname{Adj}\left(W^{T}\right) \\
\operatorname{Adj}(W) & 0
\end{array}\right)\left(\begin{array}{l}
\boldsymbol{\psi} \\
\mathbf{u}
\end{array}\right)=0 .
$$

In any quadratic line complex the lines of the complex passing through a point usually form a quadratic cone. Exceptionally, for some points this cone degenerates to a pair of planes. Such points are called the singular points of the complex. Again for a general quadratic complex the set of singular points form a degree 4 surface known as the Kummer surface, see [16]. Here the complex of acceleration lines forms a tetrahedral complex. In such a quadratic line complex the Kummer surface degenerates to a set of 4 planes. These four planes consist of the three planes $\pi_{12}, \pi_{23}$ and $\pi_{31}$, as in the previous section, together with the plane at infinity. To see this consider the lines in the complex passing through a point $\mathbf{p}$, on one of the planes. In general a line in the complex satisfies the equation,

$$
\boldsymbol{\psi}^{T} \operatorname{Adj}(W)^{T}(\mathbf{p} \times \boldsymbol{\psi})=0 .
$$

The term $(\mathbf{p} \times \boldsymbol{\psi})$ can be written as $P \boldsymbol{\psi}$, where $P$ is the $3 \times 3$ anti-symmetric matrix corresponding to the position vector $\mathbf{p}$. Then adding the equation to its transpose gives the standard form for a quadric,

$$
\boldsymbol{\psi}^{T}\left(\operatorname{Adj}(W)^{T} P-P \operatorname{Adj}(W)\right) \boldsymbol{\psi}=0 .
$$

To see that the symmetric matrix $\left(\operatorname{Adj}(W)^{T} P-P \operatorname{Adj}(W)\right)$ singular on the points of the planes $\pi_{12}, \pi_{23}$ and $\pi_{31}$ consider a point on say $\pi_{12}$, determined by $\mathbf{p}=\mu_{1} \mathbf{n}_{1}+\mu_{2} \mathbf{n}_{2}$, where $\mu_{1}$ and $\mu_{2}$ are arbitrary constants and the $\mathbf{n}_{i}$ are the eigenvectors of the angular acceleration matrix $W$. That is,

$$
W \mathbf{n}_{i}=\lambda_{i} \mathbf{n}_{i}
$$


Notice that these vectors are also eigenvectors for the matrix $\operatorname{Adj}(W)$, multiplying the above equation and rearranging gives,

$$
\operatorname{Adj}(W) \mathbf{n}_{i}=\frac{\Delta}{\lambda_{i}} \mathbf{n}_{i}
$$

where $\Delta=\operatorname{det}(W)$. The matrix is singular since there is a vector,

$$
\boldsymbol{\psi}=\mu_{1}\left(\Delta \lambda_{1}-\lambda_{1}^{2} \lambda_{2}^{2}\right) \mathbf{n}_{1}+\mu_{2}\left(\Delta \lambda_{2}-\lambda_{1}^{2} \lambda_{2}^{2}\right) \mathbf{n}_{2},
$$

that is annihilated by the matrix. Verifying this is helped by noticing that, from the above,

$$
\operatorname{Adj}(W) \boldsymbol{\psi}=\mu_{1} \Delta\left(\Delta-\lambda_{1} \lambda_{2}^{2}\right) \mathbf{n}_{1}+\mu_{2} \Delta\left(\Delta-\lambda_{1}^{2} \lambda_{2}\right) \mathbf{n}_{2},
$$

and that,

$$
\operatorname{Adj}(W)^{T}\left(\mathbf{n}_{1} \times \mathbf{n}_{2}\right)=\left(W \mathbf{n}_{1}\right) \times\left(W \mathbf{n}_{2}\right)=\lambda_{1} \lambda_{2}\left(\mathbf{n}_{1} \times \mathbf{n}_{2}\right),
$$

using (A.3).

The complex is also singular on the plane at infinity, to see this a homogenising variable $p_{0}$ must be introduced. This is multiplied by the coordinates of $\boldsymbol{\psi}$, in this way the equation for the lines of the quadric through the point $\mathbf{p}$ will be a degree 2 homogeneous equation in the coordinates of $\mathbf{p}$ and $p_{0}$. However, it is clear that the only consequence of this will be to introduce the plane of singularities at $p_{0}=0$.

\section{Points of Inflection}

Next, the velocity and acceleration of points are considered together. The point $\mathbf{c}(t)$ is an inflection if and only if its velocity vector and acceleration vector are parallel. The condition,

$$
\lambda \dot{\mathbf{c}}+\mu \ddot{\mathbf{c}}=\mathbf{0},
$$

can be written as,

$$
\lambda(\Omega \mathbf{c}+\mathbf{v})+\mu\left(W \mathbf{c}+\mathbf{a}_{0}\right)=\mathbf{0} .
$$

This is easily rearranged to give,

$$
\mathbf{c}=-(\mu W+\lambda \Omega)^{-1}\left(\mu \mathbf{a}_{0}+\lambda \mathbf{v}\right) .
$$


It is useful at this point to consider the curve $\mathbf{c}(\lambda, \mu)$ as a curve in projective space $\mathbb{P}^{3}$. Suppose that the homogeneous coordinates for this space are $(x:$ $y: z: w)$, then the curve of inflection points can be written,

$$
\left(\begin{array}{l}
x \\
y \\
z
\end{array}\right)=-\operatorname{Adj}(\mu W+\lambda \Omega)\left(\mu \mathbf{a}_{0}+\lambda \mathbf{v}\right)
$$

and

$$
w=\operatorname{det}(\mu W+\lambda \Omega)
$$

Using the results from the appendix the above equations can be expanded to give,

$$
\begin{aligned}
&\left(\begin{array}{l}
x \\
y \\
z
\end{array}\right)= \lambda^{3}(\boldsymbol{\omega} \cdot \mathbf{v}) \boldsymbol{\omega}+\lambda^{2} \mu\left((\dot{\boldsymbol{\omega}} \cdot \mathbf{v}) \boldsymbol{\omega}+(\boldsymbol{\omega} \cdot \mathbf{v}) \dot{\boldsymbol{\omega}}+\left(\boldsymbol{\omega} \cdot \mathbf{a}_{0}\right) \boldsymbol{\omega}\right)+ \\
& \lambda \mu^{2}\left((\dot{\boldsymbol{\omega}} \cdot \mathbf{v}) \dot{\boldsymbol{\omega}}+|\boldsymbol{\omega}|^{2}(\boldsymbol{\omega} \cdot \mathbf{v}) \boldsymbol{\omega}+\mathbf{v} \times(\boldsymbol{\omega} \times(\boldsymbol{\omega} \times \dot{\boldsymbol{\omega}}))+\right. \\
&\left.\left(\dot{\boldsymbol{\omega}} \cdot \mathbf{a}_{0}\right) \boldsymbol{\omega}+\left(\boldsymbol{\omega} \cdot \mathbf{a}_{0}\right) \dot{\boldsymbol{\omega}}\right)+ \\
& \mu^{3}\left(\left(\dot{\boldsymbol{\omega}} \cdot \mathbf{a}_{0}\right) \dot{\boldsymbol{\omega}}+|\boldsymbol{\omega}|^{2}\left(\boldsymbol{\omega} \cdot \mathbf{a}_{0}\right) \boldsymbol{\omega}+\mathbf{a}_{0} \times(\boldsymbol{\omega} \times(\boldsymbol{\omega} \times \dot{\boldsymbol{\omega}}))\right), \\
& w=-\mu^{3}|\boldsymbol{\omega} \times \dot{\boldsymbol{\omega}}| .
\end{aligned}
$$

This shows that the curve is a twisted cubic curve since the homogeneous coordinates are given by cubic functions in the parameters $\mu$ and $\lambda$. Moreover, the curve meets the plane at infinity when $w=0$, since $w$ is a cubic in $\mu$ the curve must osculate the plane at infinity at the point $\mu=0$. This is the defining characteristic of a cubical parabola, see [18, Chap. XII].

Returning to Euclidian space $\mathbb{R}^{3}$, we may set $s=\lambda / \mu$ so that,

$$
\mathbf{c}(s)=-(W+s \Omega)^{-1}\left(\mathbf{a}_{0}+s \mathbf{v}\right) .
$$

Notice that with this parameterisation $\mathbf{c}(0)=\mathbf{r}_{c}$, the acceleration centre. Substituting the relation for the acceleration centre; $\mathbf{a}_{0}=-W \mathbf{r}_{c}$, gives,

$$
\mathbf{c}(s)=\frac{1}{|\boldsymbol{\omega} \times \dot{\boldsymbol{\omega}}|^{2}}\left(s^{3}(\boldsymbol{\omega} \cdot \mathbf{v}) \boldsymbol{\omega}+\right.
$$




$$
\begin{aligned}
& s^{2}\left((\dot{\boldsymbol{\omega}} \cdot \mathbf{v}) \boldsymbol{\omega}+(\boldsymbol{\omega} \cdot \mathbf{v}) \dot{\boldsymbol{\omega}}-\left(\mathbf{r}_{c} \cdot(\boldsymbol{\omega} \times \dot{\boldsymbol{\omega}})\right) \boldsymbol{\omega}\right)+ \\
& s\left((\dot{\boldsymbol{\omega}} \cdot \mathbf{v}) \dot{\boldsymbol{\omega}}+|\boldsymbol{\omega}|^{2}(\boldsymbol{\omega} \cdot \mathbf{v}) \boldsymbol{\omega}+\mathbf{v} \times(\boldsymbol{\omega} \times(\boldsymbol{\omega} \times \dot{\boldsymbol{\omega}}))-\right. \\
& \left.\left.\mathbf{r}_{c} \cdot(\boldsymbol{\omega} \times(\boldsymbol{\omega} \times \dot{\boldsymbol{\omega}})) \boldsymbol{\omega}-\mathbf{r}_{c} \cdot(\boldsymbol{\omega} \times \dot{\boldsymbol{\omega}}) \dot{\boldsymbol{\omega}}\right)\right)+\mathbf{r}_{c} .
\end{aligned}
$$

This simplifies greatly if we choose to put the origin at the acceleration centre so that $\mathbf{r}_{c}=\mathbf{0}$.

$$
\begin{aligned}
\mathbf{c}(s)= & \frac{1}{|\boldsymbol{\omega} \times \dot{\boldsymbol{\omega}}|^{2}}\left(s^{3}(\boldsymbol{\omega} \cdot \mathbf{v}) \boldsymbol{\omega}+s^{2}((\dot{\boldsymbol{\omega}} \cdot \mathbf{v}) \boldsymbol{\omega}+(\boldsymbol{\omega} \cdot \mathbf{v}) \dot{\boldsymbol{\omega}})+\right. \\
& s\left((\dot{\boldsymbol{\omega}} \cdot \mathbf{v}) \dot{\boldsymbol{\omega}}+|\boldsymbol{\omega}|^{2}(\boldsymbol{\omega} \cdot \mathbf{v}) \boldsymbol{\omega}+\mathbf{v} \times(\boldsymbol{\omega} \times(\boldsymbol{\omega} \times \dot{\boldsymbol{\omega}}))\right) .
\end{aligned}
$$

Clearly if $\boldsymbol{\omega} \cdot \mathbf{v}=0$ the inflection cubic degenerates to a conic. This condition implies that the motion is instantaneously planar, since if $\boldsymbol{\omega}=0$ the motion is instantaneously a translation and if $\mathbf{v}=0$ or $\boldsymbol{\omega}$ is perpendicular to $\mathbf{v}$, the motion is instantaneously a rotation. More details on the possible degeneracy of this curve can be found in [6] and [7].

Another property of a twisted cubic curve is that it is the intersection of three quadric surfaces. An alternative condition for a point to be an inflection is that the curvature of the point's trajectory must be zero. This is because the curvature is proportional to the vector product $\dot{\mathbf{c}} \times \ddot{\mathbf{c}}$, see [17], for example. Now for an arbitrary point $\mathbf{r}$, the instantaneous velocity $\dot{\mathbf{r}}=\Omega \mathbf{r}+\mathbf{v}$ and instantaneous acceleration $\ddot{\mathbf{r}}=W \mathbf{r}+\mathbf{a}_{0}$ are linear functions of coordinates of $\mathbf{r}$. Hence the components of the vector equation,

$$
\dot{\mathbf{r}} \times \ddot{\mathbf{r}}=\mathbf{0},
$$

give three, degree 2 equations, which define 3 quadric surfaces. Clearly the inflection curve $\mathbf{c}(s)$, satisfies all three equations since points along this curve have parallel velocity and acceleration. Note that, since the cubic lies on all three quadrics it also lies on any linear combination of the quadrics, that is the cubic is the base curve for the net of quadrics.

\section{Osculating Planes}

Consider an arbitrary point $\mathbf{r}$, suppose that the osculating plane to the trajectory of this point has a normal vector $\mathbf{n}$. This normal vector will be given by the vector product,

$$
\mathbf{n}=\dot{\mathbf{r}} \times \ddot{\mathbf{r}}
$$


The set of points in space whose osculating planes are mutually parallel can be found from the solutions to the equations,

$$
\mathbf{n}_{1} \cdot \mathbf{n}=\mathbf{n}_{1} \cdot(\dot{\mathbf{r}} \times \ddot{\mathbf{r}})=0, \quad \text { and } \quad \mathbf{n}_{2} \cdot \mathbf{n}=\mathbf{n}_{2} \cdot(\dot{\mathbf{r}} \times \ddot{\mathbf{r}})=0
$$

where $\mathbf{n}_{1}$ and $\mathbf{n}_{2}$ are perpendicular to the common normal to the planes and linearly independent. Notice that each equation $\mathbf{n}_{i} \cdot(\dot{\mathbf{r}} \times \ddot{\mathbf{r}})=0$ picks out a quadric in the net of quadrics given by equation (19) above, see [18]. The intersection of a pair of such quadrics is a quartic curve, it must consist of the twisted cubic of inflections plus a line. Moreover the line will be a chord of the cubic, that is a line meeting the cubic in two points. The cubic curve here corresponds to common solutions to the two equations where $\dot{\mathbf{r}} \times \ddot{\mathbf{r}}=\mathbf{0}$, so the residual line contains the points whose osculating planes are parallel.

Any chord to a twisted cubic can be found as the residual line in the intersection of a pair of quadrics from the net defining the cubic. Moreover any point in space, not on the cubic, lies on exactly one chord of the cubic, see [18]. Hence there is a 1-to-1 correspondence between chords of the cubic of inflection points and the direction of the normal vectors to osculating planes.

This result can be proved in another way. Consider the set of points with osculating plane normal to a vector $\mathbf{n}$. These points satisfy the pair of equations,

$$
\mathbf{n} \cdot \dot{\mathbf{r}}=0, \quad \text { and } \quad \mathbf{n} \cdot \ddot{\mathbf{r}}=0 .
$$

Substituting for the velocity and acceleration vectors gives a pair of linear equations in the position $\mathbf{r}$,

$$
\mathbf{n}^{T} \Omega \mathbf{r}+\mathbf{n}^{T} \mathbf{v}=0, \quad \mathbf{n}^{T} W \mathbf{r}+\mathbf{n}^{T} \mathbf{a}_{0}=0
$$

Linear equations define planes in space, the normals to these planes are given by, $\mathbf{m}_{1}=\Omega^{T} \mathbf{n}=\mathbf{n} \times \boldsymbol{\omega}$ and $\mathbf{m}_{2}=W^{T} \mathbf{n}$. The intersection of the two planes is a line in general. The direction of this line of points with parallel osculating planes will be given by $\mathbf{m}_{1} \times \mathbf{m}_{2}$. Further, the points on the line can be parameterised as,

$$
\mathbf{r}(\mu)=\mathbf{r}_{0}+\mu\left(\mathbf{m}_{1} \times \mathbf{m}_{2}\right),
$$

where $\mathbf{r}_{0}$ is a given point on the line and $\mu$ an arbitrary parameter. The normal to the osculating plane for any point on this line will be some multiple of $\mathbf{n}$ by hypothesis, this can be written,

$$
\kappa(\mu) \mathbf{n}=\dot{\mathbf{r}} \times \ddot{\mathbf{r}}=(\Omega \mathbf{r}(\mu)+\mathbf{v}) \times\left(W \mathbf{r}(\mu)+\mathbf{a}_{0}\right),
$$


where $\kappa(\mu)$ is a multiplicative factor depending on the parameter $\mu$. Substituting from (20) gives,

$$
\begin{gathered}
\kappa(\mu) \mathbf{n}=\dot{\mathbf{r}}_{0} \times \ddot{\mathbf{r}}_{0}+\mu\left(\Omega\left(\mathbf{m}_{1} \times \mathbf{m}_{2}\right) \times \ddot{\mathbf{r}}_{0}-W\left(\mathbf{m}_{1} \times \mathbf{m}_{2}\right) \times \dot{\mathbf{r}}_{0}\right)+ \\
\mu^{2}\left(\Omega\left(\mathbf{m}_{1} \times \mathbf{m}_{2}\right) \times W\left(\mathbf{m}_{1} \times \mathbf{m}_{2}\right)\right) .
\end{gathered}
$$

Notice that since $\dot{\mathbf{r}}_{0}, \ddot{\mathbf{r}}_{0}, \Omega\left(\mathbf{m}_{1} \times \mathbf{m}_{2}\right)$ and $W\left(\mathbf{m}_{1} \times \mathbf{m}_{2}\right)$ are coplanar, the vector products on the right-hand side of the above equation are all parallel to $\mathbf{n}$ so $\kappa(\mu)$ is given by a quadratic in $\mu$. The root of this quadratic are points where the instantaneous velocity and instantaneous acceleration are collinear, that is inflection points. Hence this line meets the cubic of inflection points at two points, the roots of the quadratic, and hence is a chord of the cubic curve.

On the other hand, suppose that $\mathbf{c}_{1}$ and $\mathbf{c}_{2}$ are two points on the cubic of inflection points, the line joining these points, a chord to the cubic, can be parameterised as,

$$
\mathbf{r}(\lambda)=(1-\lambda) \mathbf{c}_{1}+\lambda \mathbf{c}_{2}
$$

At any point on this line the normal to the osculating plane is given by,

$$
\dot{\mathbf{r}}(\lambda) \times \ddot{\mathbf{r}}(\lambda)=\lambda(1-\lambda)\left(\dot{\mathbf{c}}_{1} \times \ddot{\mathbf{c}}_{2}+\dot{\mathbf{c}}_{2} \times \ddot{\mathbf{c}}_{1}\right) .
$$

The terms, $\dot{\mathbf{c}}_{1} \times \ddot{\mathbf{c}}_{1}$ and $\dot{\mathbf{c}}_{2} \times \ddot{\mathbf{c}}_{2}$ vanish since $\mathbf{c}_{1}$ and $\mathbf{c}_{2}$ are inflection points. In the equation above the direction of the normal is independent of the parameter $\lambda$ and hence is constant along the chord. In particular notice that the direction of the normal to the osculating planes along a chord is given by,

$$
\mathbf{n}=\left(\dot{\mathbf{c}}_{1} \times \ddot{\mathbf{c}}_{2}+\dot{\mathbf{c}}_{2} \times \ddot{\mathbf{c}}_{1}\right)
$$

where $\mathbf{c}_{1}$ and $\mathbf{c}_{2}$ are the points where the chord meets the cubic of inflection points. Of course the magnitude of the normal vector is arbitrary in the above equation. The equation can be simplified further by recalling that for points of inflection $\mathbf{c}_{1}$ and $\mathbf{c}_{2}$ the velocities and acceleration are parallel and satisfy (15) above. Hence ignoring the magnitude of the normal vector again we have,

$$
\mathbf{n}=\dot{\mathbf{c}}_{1} \times \dot{\mathbf{c}}_{2}
$$

\section{The Linear Complex of Tangent Lines}

The methods of the previous section can be extended to study the tangents to the cubic of inflection points. These tangents are, of course, also 
chords to the curve. Suppose that $\mathbf{c}$ is a point on the cubic of inflection points and $\mathbf{t}$ is the direction of the tangent line at this point. The tangent line will be given by the parameterisation,

$$
\mathbf{r}(\lambda)=\mathbf{c}+\lambda \mathbf{t}
$$

Notice that, with this parameterisation,

$$
\dot{\mathbf{r}}(\lambda)=\Omega \mathbf{c}+\lambda \Omega \mathbf{t}+\mathbf{v}=\dot{\mathbf{c}}+\lambda \Omega \mathbf{t},
$$

and similarly,

$$
\ddot{\mathbf{r}}(\lambda)=W \mathbf{c}+\lambda W \mathbf{t}+\mathbf{a}_{0}=\ddot{\mathbf{c}}+\lambda W \mathbf{t} .
$$

So the normal to the osculating plane to points along this line will be given by,

$$
\dot{\mathbf{r}}(\lambda) \times \ddot{\mathbf{r}}(\lambda)=\lambda(\Omega \mathbf{t} \times \ddot{\mathbf{c}}+\dot{\mathbf{c}} \times W \mathbf{t})+\lambda^{2}(\Omega \mathbf{t}) \times(W \mathbf{t}) .
$$

In order that $\mathbf{t}$ is the tangent direction the tangent line must meet the inflection cubic at a single point, this is only possible if the $\lambda$ term in the above equation is zero. This gives a vector equation for the tangent direction,

$$
\Omega \mathbf{t} \times \ddot{\mathbf{c}}+\dot{\mathbf{c}} \times W \mathbf{t}=\mathbf{0} .
$$

Now since $\mathbf{c}$ is an inflection point $\dot{\mathbf{c}}$ and $\ddot{\mathbf{c}}$ are parallel and indeed from (15) above we have that $\ddot{\mathbf{c}}=-s \dot{\mathbf{c}}$. Substituting this relation and rearranging the above equation gives,

$$
\dot{\mathbf{c}} \times(W+s \Omega) \mathbf{t}=\mathbf{0} .
$$

The vector product of two non-zero vectors will only vanish if the vectors are parallel so we can infer that $\dot{\mathbf{c}}$ and $(W+s \Omega) \mathbf{t}$ are parallel and so we may set,

$$
\mathbf{t}=(W+s \Omega)^{-1} \dot{\mathbf{c}} .
$$

This result can also be found by differentiating the results at the end of section 7 with respect to the parameter $s$.

Another known property of twisted cubic curves is that the tangent lines to the curve lie in a unique linear line complex, [19]. Here we look at the linear line complex determined by the cubical parabola of inflection points. Historically, linear line complexes were referred to as screws. This is because a linear line complex is uniquely determined by a screw. A twist is an element of the Lie algebra to the group of rigid transformations, se(3). A screw is 
an element of the projectivisation of this Lie algebra, that is a ray through the origin in $s e(3)$, or to put it yet another way a screw is represented by a twist but multiplying the twist by any non-zero constant represents the same screw.

So a twisted cubic curve determines a unique screw. To see this we can use a parameterisation of the curve given by,

$$
\mathbf{c}(s)=\mathbf{k}_{3} s^{3}+\mathbf{k}_{2} s^{2}+\mathbf{k}_{1} s .
$$

Comparing this with the result given in (18) above, it can be seen that,

$$
\begin{aligned}
& \mathbf{k}_{3}=\frac{1}{|\boldsymbol{\omega} \times \dot{\boldsymbol{\omega}}|^{2}}(\boldsymbol{\omega} \cdot \mathbf{v}) \boldsymbol{\omega}, \\
& \mathbf{k}_{2}=\frac{1}{|\boldsymbol{\omega} \times \dot{\boldsymbol{\omega}}|^{2}}((\dot{\boldsymbol{\omega}} \cdot \mathbf{v}) \boldsymbol{\omega}+(\boldsymbol{\omega} \cdot \mathbf{v}) \dot{\boldsymbol{\omega}}), \\
& \mathbf{k}_{1}=\frac{1}{|\boldsymbol{\omega} \times \dot{\boldsymbol{\omega}}|^{2}}\left((\dot{\boldsymbol{\omega}} \cdot \mathbf{v}) \dot{\boldsymbol{\omega}}+|\boldsymbol{\omega}|^{2}(\boldsymbol{\omega} \cdot \mathbf{v}) \boldsymbol{\omega}+\mathbf{v} \times(\boldsymbol{\omega} \times(\boldsymbol{\omega} \times \dot{\boldsymbol{\omega}})) .\right.
\end{aligned}
$$

On the other hand, notice that the vectors $\mathbf{k}_{i}$ can be found by differentiating $\mathbf{c}(s)$ with respect to $s$ and then setting $s$ to 0 . In particular we have that $\mathbf{k}_{1}=\mathbf{c}^{\prime}(0)=-W^{-1} \mathbf{v}=\mathbf{t}_{0}$. Where $\mathbf{t}_{0}$ denotes the tangent vector at the acceleration centre. Continuing in this fashion produces, $\mathbf{k}_{2}=(1 / 2) \mathbf{c}^{\prime \prime}(0)=$ $W^{-1} \Omega W^{-1} \mathbf{v}=-W^{-1} \Omega \mathbf{t}_{0}$ and $\mathbf{k}_{3}=(1 / 6) \mathbf{c}^{(3)}(0)=-\left(W^{-1} \Omega\right)^{2} W^{-1} \mathbf{v}=$ $\left(W^{-1} \Omega\right)^{2} \mathbf{t}_{0}$.

Returning to the tangent lines to the curve, these are given in Plücker coordinates by,

$$
\left(\begin{array}{c}
\mathbf{c}^{\prime}(s) \\
\mathbf{c}(s) \times \mathbf{c}^{\prime}(s)
\end{array}\right)=\left(\begin{array}{c}
3 \mathbf{k}_{3} s^{2}+2 \mathbf{k}_{2} s+\mathbf{k}_{1} \\
\left(\mathbf{k}_{2} \times \mathbf{k}_{3}\right) s^{4}+2\left(\mathbf{k}_{1} \times \mathbf{k}_{3}\right) s^{3}+\left(\mathbf{k}_{1} \times \mathbf{k}_{2}\right) s^{2}
\end{array}\right)
$$

where, as above, the dash indicates differentiation with respect to the parameter $s$.

Now suppose that $\boldsymbol{\zeta}(s)^{T}=\left(\boldsymbol{\psi}^{T}, \mathbf{u}^{T}\right)$ is an arbitrary twist, if this is reciprocal to the tangent lines of the inflection cubic it must satisfy,

$$
\left(3 \mathbf{k}_{3} s^{2}+2 \mathbf{k}_{2} s+\mathbf{k}_{1}\right) \cdot \mathbf{u}+\left(\left(\mathbf{k}_{2} \times \mathbf{k}_{3}\right) s^{4}+2\left(\mathbf{k}_{1} \times \mathbf{k}_{3}\right) s^{3}+\left(\mathbf{k}_{1} \times \mathbf{k}_{2}\right) s^{2}\right) \cdot \boldsymbol{\psi}=0
$$

This must be satisfied for all $s$, by inspection the solution is,

$$
\boldsymbol{\psi}=3 \mathbf{k}_{3}, \quad \mathbf{u}=\mathbf{k}_{2} \times \mathbf{k}_{1}
$$


or any constant multiple of this. As a partitioned 6-vector this can be written in several ways,

$$
\boldsymbol{\zeta}=\left(\begin{array}{c}
\mathbf{c}^{(3)}(0) \\
\mathbf{c}^{\prime \prime}(0) \times \mathbf{c}^{\prime}(0)
\end{array}\right)=\left(\begin{array}{c}
(1 / 2)\left(W^{-1} \Omega\right)^{2} \mathbf{t}_{0} \\
\mathbf{t}_{0} \times\left(W^{-1} \Omega\right) \mathbf{t}_{0}
\end{array}\right) .
$$

This expression could be expanded in terms of the angular velocity and angular acceleration of the body, this will produce large expressions which do not seem to be very illuminating. However, it is worth noting that the direction of the axis of this screw is parallel to the direction of the angular velocity of the body $\boldsymbol{\omega}$, and the moment is directed along the binormal to the curve of inflection points at the acceleration centre.

\section{The Bresse Hyperboloid}

Consider the points in the moving body whose velocity and acceleration vectors are instantaneously perpendicular. From the above, such point will satisfy the equation,

$$
\dot{\mathbf{r}} \cdot \ddot{\mathbf{r}}=(\Omega \mathbf{r}+\mathbf{v})^{T}\left(W \mathbf{r}+\mathbf{a}_{0}\right)=0 .
$$

Expanding this equation and adding it to its transpose it is possible to rewrite it in matrix form as,

$$
\left(\mathbf{r}^{T}, 1\right)\left(\begin{array}{cc}
\Omega^{T} W+W^{T} \Omega & \Omega^{T} \mathbf{a}_{0}+W^{T} \mathbf{v} \\
\mathbf{v}^{T} W+\mathbf{a}_{0}^{T} \Omega & 2 \mathbf{a}_{0}^{T} \mathbf{v}
\end{array}\right)\left(\begin{array}{l}
\mathbf{r} \\
1
\end{array}\right)=0 .
$$

This represents a quadric surface, it is usually known as the Bresse hyperboloid, see [13]. To study this surface in more detail first notice that the top left-hand block simplifies:

$$
\Omega^{T} W+W^{T} \Omega=-\Omega\left(\dot{\Omega}+\Omega^{2}\right)+\left(-\dot{\Omega}+\Omega^{2}\right) \Omega=-(\Omega \dot{\Omega}+\dot{\Omega} \Omega),
$$

as do the off-diagonal blocks:

$$
\Omega^{T} \mathbf{a}_{0}+W^{T} \mathbf{v}=\Omega^{T}(\dot{\mathbf{v}}+\boldsymbol{\omega} \times \mathbf{v})+\left(\dot{\Omega}+\Omega^{2}\right)^{T} \mathbf{v}=\dot{\mathbf{v}} \times \boldsymbol{\omega}-\dot{\boldsymbol{\omega}} \times \mathbf{v},
$$

using the definitions $W=\left(\dot{\Omega}+\Omega^{2}\right)$ and $\mathbf{a}_{0}=\dot{\mathbf{v}}+\boldsymbol{\omega} \times \mathbf{v}$.

Next it is possible to find a point where the bottom left and top righthand blocks of the matrix vanish. Since the matrix is symmetric, only one 
of these blocks needs to be considered, suppose the point sought is denoted $\mathbf{r}_{B}$ then it can be found from the equation,

$$
\left(\Omega^{T} W+W^{T} \Omega\right) \mathbf{r}_{B}+\Omega^{T} \mathbf{a}_{0}+W^{T} \mathbf{v}=\mathbf{0},
$$

or using the relation in equations (28) and (29) and solving for $\mathbf{r}_{B}$,

$$
\mathbf{r}_{B}=(\Omega \dot{\Omega}+\dot{\Omega} \Omega)^{-1}(\dot{\mathbf{v}} \times \boldsymbol{\omega}-\dot{\boldsymbol{\omega}} \times \mathbf{v}) .
$$

This point is, of course, the centre of the hyperboloid. Now if coordinates are chosen so that $\mathbf{r}_{B}$ is at the origin, the matrix equation for the hyperboloid becomes,

$$
\left(\mathbf{r}^{T}, 1\right)\left(\begin{array}{cc}
-(\Omega \dot{\Omega}+\dot{\Omega} \Omega) & \mathbf{0} \\
\mathbf{0}^{T} & 2 \mathbf{v}^{T} \dot{\mathbf{v}}
\end{array}\right)\left(\begin{array}{l}
\mathbf{r} \\
1
\end{array}\right)=0,
$$

where the bottom right entry has been simplified using the definition of $\mathbf{a}_{0}$ again.

Having chosen the position of the origin of coordinates the orientation can be chosen as in section 4 above. That is, the $x$-axis is chosen to be parallel to the angular velocity vector, so that $\boldsymbol{\omega}=(\omega, 0,0)^{T}$. The $x y$-plane is chosen to contain the angular acceleration vector, so $\dot{\boldsymbol{\omega}}=(\alpha \cos \phi, \alpha \sin \phi, 0)^{T}$, where $\alpha=|\dot{\boldsymbol{\omega}}|$ and $\omega=|\boldsymbol{\omega}|$. With the coordinate axes oriented in this way the $4 \times 4$ symmetric matrix in (32) becomes,

$$
Q_{B}=\left(\begin{array}{cccc}
0 & -\alpha \omega \sin \phi & 0 & 0 \\
-\alpha \omega \sin \phi & 2 \alpha \omega \cos \phi & 0 & 0 \\
0 & 0 & 2 \alpha \omega \cos \phi & 0 \\
0 & 0 & 0 & 2 \mathbf{v}^{T} \dot{\mathbf{v}}
\end{array}\right)
$$

The signs of the eigenvalues of $Q_{B}$ reveal the geometrical nature of the quadric surface determined by this matrix. The eigenvalues are simple to compute,

$$
\lambda_{1}=2 \alpha \omega \cos \phi, \lambda_{2}=\alpha \omega(\cos \phi+1), \lambda_{3}=\alpha \omega(\cos \phi-1) \quad \text { and } \quad \lambda_{4}=2 \mathbf{v}^{T} \dot{\mathbf{v}} .
$$

Now it is easy to see that $\lambda_{2}$ is non-negative and $\lambda_{3}$ is non-positive, hence if $\lambda_{1}$ and $\lambda_{4}$ have opposite signs then the surface is a hyperboloid of one sheet. While if $\lambda_{1}$ and $\lambda_{4}$ have the same sign then the surface is a hyperboloid of two sheets. This can be summarised neatly as,

$$
\text { if }(\boldsymbol{\omega} \cdot \dot{\boldsymbol{\omega}})(\mathbf{v} \cdot \dot{\mathbf{v}})= \begin{cases}>0, & \text { two sheets } \\ <0, & \text { one sheet. }\end{cases}
$$


When $(\boldsymbol{\omega} \cdot \dot{\boldsymbol{\omega}})(\mathbf{v} \cdot \dot{\mathbf{v}})=0$ the situation is more complicated. Certainly when $(\mathbf{v} \cdot \dot{\mathbf{v}})=0$, the Bresse hyperboloid degenerates to a cone with vertex at $\mathbf{r}_{B}$. However, if $(\boldsymbol{\omega} \cdot \dot{\boldsymbol{\omega}})=0$, then the matrix $(\Omega \dot{\Omega}+\dot{\Omega} \Omega)$ is singular and hence it is not possible to solve for $\mathbf{r}_{B}$. The matrix $Q_{B}$, however, will not usually be singular and hence when $\boldsymbol{\omega}$ and $\dot{\boldsymbol{\omega}}$ are perpendicular the Bresse hyperboloid will be a non-central quadric surface. Not a hyperboloid but a paraboloid.

If $\boldsymbol{\omega}$ and $\dot{\boldsymbol{\omega}}$ are parallel, $(\Omega \dot{\Omega}+\dot{\Omega} \Omega)$ is singular but it is straightforward to see that the Bresse hyperboloid degenerates to a cylinder, that is a cone with vertex at infinity. When either $\boldsymbol{\omega}$ or $\dot{\boldsymbol{\omega}}$ vanish, then the Bresse hyperboloid degenerates to a repeated plane.

\section{Conclusions}

In this work no mention has been made of axodes nor of any analogue of the Euler-Savary equation of planar kinematics. This is not seen as a disadvantage, but just as an indication that these ideas are not central in the subject. Rather, they are interesting objects, which may merit investigation in their own right. In particular, this work only studies the effects of spatial motion on points. A similar study could be carried out for lines in space. Given a line, a spatial motion will determine a one parameter family of lines, that is a ruled surface, by sweeping the original given line. The local properties of such surfaces could be related to the properties of the motion. For example the set of lines that are instantaneously moving in a plane lie in a quadratic line complex, this is a simple first order property of the motion.

This work only looks at properties of spatial motion up to second order. Of course it is possible, but harder, to look at properties up-to third order and higher. This is a less well understood area although some results are known. For example, it is well known that in a general spatial motion the point in space that are at points of zero torsion in their trajectories form a cubic surface.

A less explored direction here is the connection with special motions. For example, if it is known that a motion is say line symmetric, what does that imply about the acceleration ellipsoids? This problem could also be approached the other way around. Consider the circumstances where, for example there is a single, thrice repeated, acceleration axis. The conditions for this to happen can be thought of as differential equations for the twist of the motion. The question now is: can these differential equations be 
integrated? If the answer is yes, then the result will be a new special motion for which the acceleration axes have this property at every instant. Notice there is already a nice example of this. Consider the cubic of zero-torsion points in space, under some circumstances this can degenerate in such a way that every point in space has zero torsion, see [6] and [7]. The conditions under which this occurs must be integrable, since it is known that there is a special motion under which each point moves on a plane, that is the Darboux motion.

Although this is an old and well researched area, there remains a lot of scope for further work.

\section{Acknowledgment}

I am grateful to P. Cardou for reading and commenting on a draft version of this work. I would also like to thank the reviewers whose helpful comments have also improved this work.

\section{References}

[1] Cardou, P., and Angeles, J., (2008), Estimating the Angular Velocity of a Rigid Body Moving in the Plane from Tangential and Centripetal Acceleration Measurements, Multibody System Dynamics, vol. 19, no. 4, pp. 383-406.

[2] Bottema, O. and Roth, B. (1990), Theoretical Kinematics, Dover Publications, New York.

[3] Skreiner, M. (1966), A Study of the Geometry and the Kinematics of Instantaneous Spatial Motion, J. Mecanisms vol. 1, pp. 115-143.

[4] De Lun Wang, Jian Liu and Da Zhun Xiao, (1997), Kinematic differential geometry of a rigid body in spatial motionI. A new adjoint approach and instantaneous properties of a point trajectory in spatial kinematics, Mechanism and Machine Theory vol. 32, no. 4, pp 419-432.

[5] Mohamed, M.G. (1997), Kinematics of rigid bodies in general spatial motion: second-order motion properties, Applied Mathematical Modelling, vol. 21, no. 8, pp. 471-479. 
[6] Veldkamp, G.R. (1967), Canonical Systems and Instantaneous Invariants in Spatial Kinematics, J. Mechanisms., vol. 3, no. 3, pp. 329-388.

[7] Chapman, S.C. (1995), The Geometry of the Point-Paths Generated by Rigid-Body Motions in Two and Three Dimensions, M.Sc. Thesis submitted to Victoria University of Wellington, NZ.

[8] Martínez, J.M.R. and Duffy, J. (1998), Determination of the acceleration center of a rigid body in spatial motion, European Journal of Mechanics - A/Solids vol. 17, no. 6, pp. 969-977.

[9] Bokelberg, E.H., Hunt, K.H., and Ridley, P.R. (1992), Spatial Motion I Points of Inflection and the Differential Geometry of Screws, Mech. Mach. Theory, vol. 27, no. 1, pp. 1-15.

[10] Angeles, J. (1999), The angular-acceleration tensor of rigid-body kinematics and its properties, Archive of Applied Mechanics, vol. 69, no. 3, pp. 204-214.

[11] Everett, J.D. (1875), On the Kinematics of a Rigid Body, Q. J. Pure Applied Math., vol. 13, pp. 33-66.

[12] Skreiner, M. (1967), On the points of inflection in general spatial motion, J. Mechanisms vol. 2, no. 4, pp. 429-433.

[13] Ridley, P.R., Bokelberg, E.H. and Hunt, K.H. (1992), Spatial Motion II Acceleration and the Differential Geometry of Screws, Mech. Mach. Theory, vol. 27, no. 1, pp. 17-35.

[14] J.M. Selig and Y. Wu, Interpolated Rigid-Body Motions and Robotics, IEEE International Conference on Robotics and Automation, Beijing 2006, pp. 1086-1091.

[15] Weisstein, Eric W. "Polynomial Discriminant." From MathWorld - A Wolfram Web Resource. http://mathworld.wolfram.com/PolynomialDiscriminant.html (Last visit $6 / 2 / 2009)$.

[16] C.M. Jessop, A Treatise on the Line Complex, Cambridge University Press, Cambridge, 1903. 
[17] Marsh, D., (2005), Applied Geometry for Computer Graphics and CAD, 2nd edition London, Springer Verlag.

[18] Semple, J.G. and Kneebone, G.T. (1952), Algebraic Projective Geometry, Oxford, Oxford University Press. (Reissued 1998).

[19] Wood, P.W. (1913) The Twisted Cubic, Cambridge Tracts in Mathematics and Mathematical Physics vol. 14, Cambridge University Press, Cambridge.

\section{Appendix A. Some Relations for $3 \times 3$ Matrices}

Several small relations concerning $3 \times 3$ matrices are collected together here.

Suppose that $A$ and $B$ are $3 \times 3$ anti-symmetric matrices corresponding to vectors $\mathbf{a}=\left(a_{x}, a_{y}, a_{z}\right)^{T}$ and $\mathbf{b}=\left(b_{x}, b_{y}, b_{z}\right)^{T}$. That is,

$$
A=\left(\begin{array}{ccc}
0 & -a_{z} & a_{y} \\
a_{z} & 0 & -a_{x} \\
-a_{y} & a_{x} & 0
\end{array}\right)
$$

and similar for $B$. So that for an arbitrary vector $\mathbf{r}, A \mathbf{r}=\mathbf{a} \times \mathbf{r}$. Now let $\lambda$ and $\mu$ be constants, then

$$
\operatorname{det}\left(\lambda A+\mu B^{2}\right)=-\lambda^{2} \mu|\mathbf{a} \times \mathbf{b}|^{2} .
$$

To show this, rotate the coordinates so that $\mathbf{b}$ is aligned with the $z$-axis. In these coordinates the matrices become,

$$
A^{\prime}=R A R^{T}, \quad\left(B^{\prime}\right)^{2}=R B^{2} R^{T}=\left(\begin{array}{ccc}
-b_{z}^{\prime 2} & 0 & 0 \\
0 & -b_{z}^{\prime 2} & 0 \\
0 & 0 & 0
\end{array}\right) .
$$

So that,

$$
\operatorname{det}\left(\lambda A+\mu B^{2}\right)=\operatorname{det}\left(\lambda A^{\prime}+\mu\left(B^{\prime}\right)^{2}\right)=\operatorname{det}\left(\begin{array}{ccc}
-\mu b_{z}^{\prime 2} & -\lambda a_{z}^{\prime} & \lambda a_{y}^{\prime} \\
\lambda a_{z}^{\prime} & -\mu b_{z}^{\prime 2} & -\lambda a_{x}^{\prime} \\
-\lambda a_{y}^{\prime} & \lambda a_{x}^{\prime} & 0
\end{array}\right) .
$$

Evaluating the determinant gives, $-\lambda^{2} \mu\left(a_{x}^{\prime 2}+a_{y}^{\prime 2}\right) b_{z}^{\prime 2}$. In terms of vector and scalar products this can be written as $-\lambda^{2} \mu\left|\mathbf{a}^{\prime} \times \mathbf{b}^{\prime}\right|^{2}$ and finally, the transformation properties of the vector and scalar products give the result. 
The second useful result concerns the adjugate matrix. Recall that for an non-singular matrix $X$, its adjugate $\operatorname{Adj}(X)$, satisfies, $\operatorname{Adj}(X)=\operatorname{det}(X) X^{-1}$. Here we have the result,

$$
\operatorname{Adj}\left(A+B^{2}\right)=\left(A+B^{2}\right)^{2}+2|\mathbf{b}|^{2}\left(A+B^{2}\right)+\left(|\mathbf{a}|^{2}+|\mathbf{b}|^{4}\right) I .
$$

This result can be found in the same way as the previous one, computing the result in a particular coordinate system and then expressing the result using invariants. Alternatively, the Cayley-Hamilton theorem could be used to find a polynomial satisfied by the matrix $\left(A+B^{2}\right)$. This equation could then be multiplied by $\left(A+B^{2}\right)^{-1}$ and rearranged to produce the result.

In either case the following simple result, valid for any $3 \times 3$ anti-symmetric matrix $A$,

$$
A^{2}=\mathbf{a a}^{T}-|\mathbf{a}|^{2} I,
$$

can be used to rewrite the equation in terms of vectors as,

$$
\operatorname{Adj}\left(A+B^{2}\right)=\mathbf{a a}^{T}+(\mathbf{a} \times \mathbf{b}) \mathbf{b}^{T}-\mathbf{b}(\mathbf{a} \times \mathbf{b})^{T}+|\mathbf{b}|^{2} \mathbf{b} \mathbf{b}^{T} .
$$

Finally, suppose $M$ is a non-singular $3 \times 3$ matrix and $\mathbf{u}, \mathbf{v}$ are 3 -vectors, then

$$
(M \mathbf{u}) \times(M \mathbf{v})=\operatorname{Adj}(M)^{T}(\mathbf{u} \times \mathbf{v}) .
$$

To see this let $\mathbf{w}$ be another arbitrary 3 -vector. Now

$$
\mathbf{w} \cdot(\mathbf{u} \times \mathbf{v})=\operatorname{det}(\mathbf{w}|\mathbf{u}| \mathbf{v}),
$$

that is the scalar triple product is the determinant of the $3 \times 3$ matrix whose columns are the 3 vectors. Multiplying by the non-singular matrix $M$ gives,

$$
\begin{aligned}
(M \mathbf{w}) \cdot((M \mathbf{u}) \times(M \mathbf{v})) & =\operatorname{det}((M \mathbf{w})|(M \mathbf{u})|(M \mathbf{v})) \\
& =\operatorname{det}(M(\mathbf{w}|\mathbf{u}| \mathbf{v})) \\
& =\operatorname{det}(M) \operatorname{det}(\mathbf{w}|\mathbf{u}| \mathbf{v}) \\
& =\operatorname{det}(M)(\mathbf{w} \cdot(\mathbf{u} \times \mathbf{v}))
\end{aligned}
$$

Now the determinant can be written as $\operatorname{det}(M)=\operatorname{Adj}(M) M=M^{T} \operatorname{Adj}(M)^{T}$ and the scalar product can be written as a matrix product to give,

$$
\mathbf{w}^{T} M^{T}(M \mathbf{u}) \times(M \mathbf{v})=\mathbf{w}^{T} M^{T} \operatorname{Adj}(M)^{T}(\mathbf{u} \times \mathbf{v}) .
$$

Since $\mathbf{w}$ and $M$ are arbitrary they can be cancelled to give the result above. 